\title{
MRS Issues Statement on MS\&E Study Report
}

Speaking on behalf of the Materials Research Society, 1989 MRS President R.P.H. Chang announced in December that

"The Materials Research Society applauds the study report on Materials Science and Engineering recently released by the National Research Council for the National Academies. MRS believes that this study succinctly captures the needs and opportunities of the field for the $1990 \mathrm{~s}$ and hopes broad support from the materials community is forthcoming to aid in the implementation of study recommendations."
President Chang went on to urge all materials-related technical societies to take an active role on behalf of the community in pursuing implementation.

Expanding on the Society statement, Kathleen C. Taylor, chair of the MRS External Affairs Committee, said that the roles of technical societies "should be both individual and collective. Individual roles would include traditional activities such as in the area of continuing education. For MRS, in particular, continuing education means specialized instruction in emerging areas of the field."

She further stated that another tradi- tional activity is the facilitation of communication through meetings and publications. All of the available, most effective communication media should be exploited. At its recent Fall meeting, for example, MRS took a first step with a satellite broadcast of its Symposium $X$, a series of overview lectures for the nonspecialist in several frontier areas of materials research. Many other opportunities exist for which a more effective role for the technical societies would be collective or cooperative.

"To bring the full breadth and excitement of our field to the educational challenge and to demonstrate to policymakers the vital role of materials science and engineering for the national welfare, societies must coordinate and pool their efforts in order to be effective," Taylor emphasized.

"The Materials Research Society is eager to work with other societies toward the formidable changes required to put the recommendations of the MS\&E Study in place."
MKS 122 Baratron

4 decades of pressure measurement

independent of gas composition

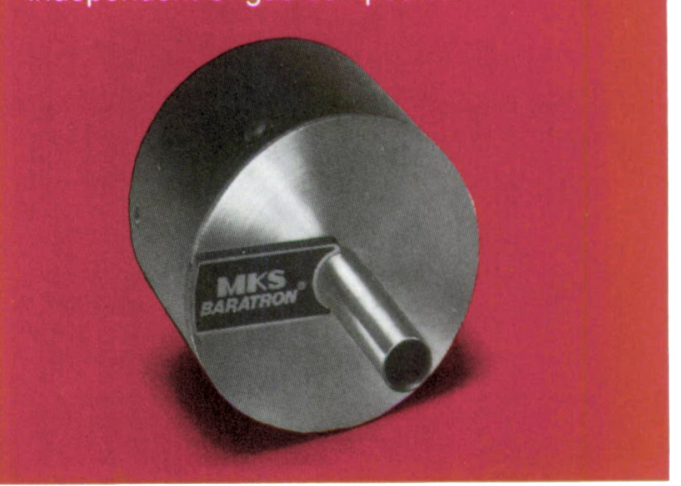

1259 Mass-Flo controller

fast response, $\pm 0.8 \%$ accuracy

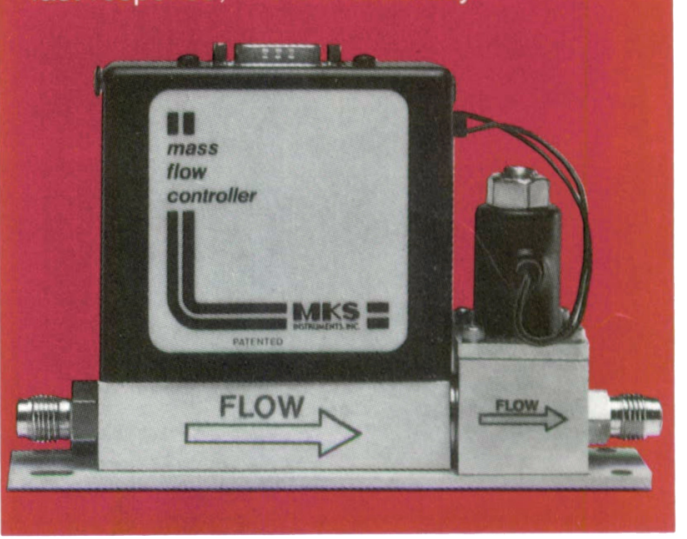




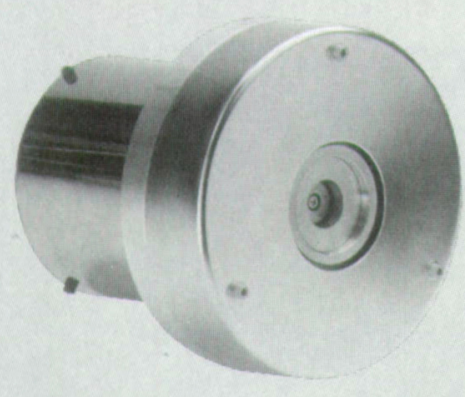

Research S-Gun

The most flexible source for research applications

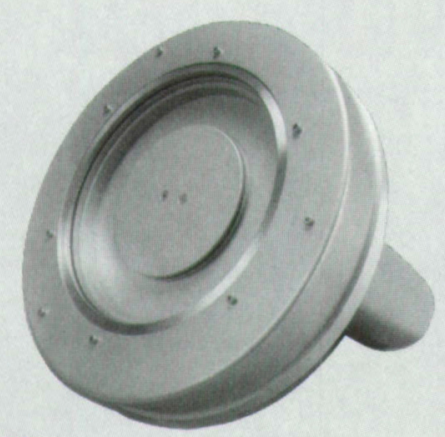

PSG-7 S-Gun

Coats 4" wafers - capable of RF, DC and Reactive Sputtering.
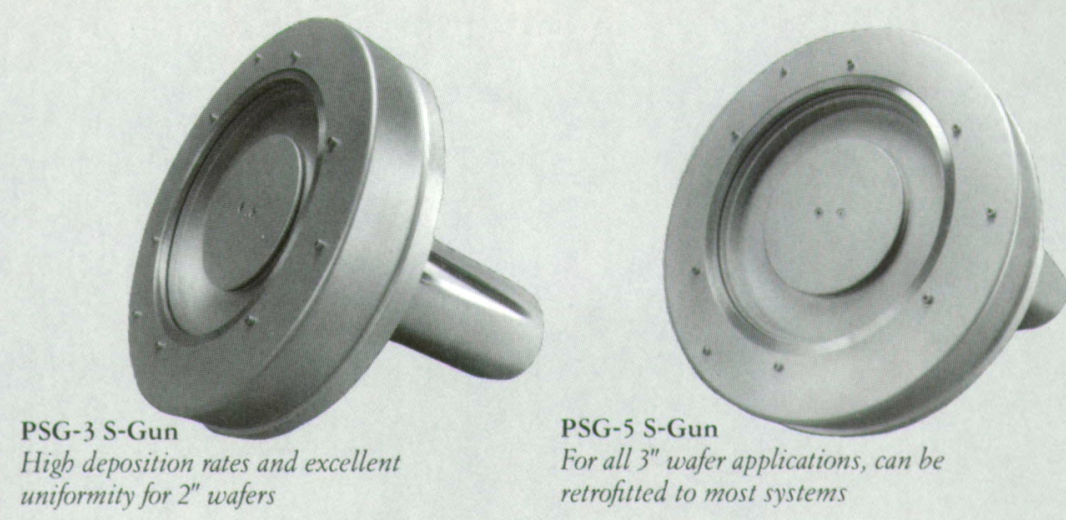

retrofitted to most systems

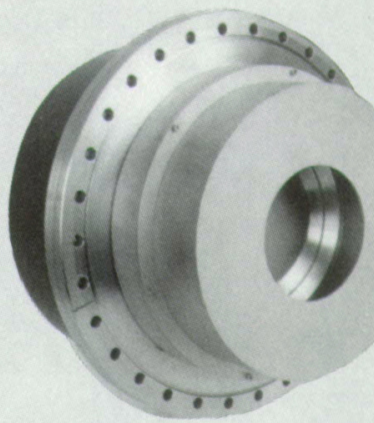

UHV S-Gun

For Ultra-High-Vacuum Applications, our UHV S-Gun is available in various sizes

\section{Now there are 6 ways to}

increase thin film reliability.

\section{Sputtered Films invented the world's most dependable deposition source.}

If you're looking to deposit more reliable thin films, look to Sputtered Films. Our coaxial magnetron S-Gun has been recognized as the world's most reliable sputter deposition source. + Consider the advantages of our coaxial magnetron design:

\section{Highest power density magnetron} The S-Gun design is the highest power density magnetron available, permitting high deposition rates and excellent film uniformity with a wide variety of materials. Even more difficult materials such as metals that form tenacious oxides can be easily sputtered with an S-Gun.

\section{Central anode}

The S-Gun design employs an effective central anode, a feature you won't find on any other magnetron source. Deposition performance is enhanced because electrons and negative ions go to ground through the anode during the sputtering process. $\neq$ And since the anode can be biased, depositions at high rates can be accomplished without subjecting substrates to excessive heat.

\section{Constant voltage,} current and rate

Uniformity characteristics of the S-Gun are excellent. That's because voltage, current and rate are held virtually constant over the life of the cathode, assuring consistent deposition of every wafer coated. We urge you to compare S-Gun performance to any other deposition source.

Quick target changes and improved utilization Approximately $45 \%$ of a target can be sputtered before replacement is necessary. The balance is totally reclaimable. Target changes take only 5 minutes because the sputter cathode slips directly into the cooling water jacket. No bonding plates are necessary.

\section{Easy to maintain}

All S-Guns are designed for effective maintenance; inhouse personnel can handle all routine service.

\section{†VLSI Research, Inc. 1988}

$¥$ See technical papers by Cumo of IBM and Clarke of SFI for discussion of negative ion effect during reactive sputtering of oxides.

For more information contact:

Sputtered Films, Inc.

314 Edison Ave.

Santa Barbara, CA 93103

Phone 805/963-9651

Fax 805/963-2959

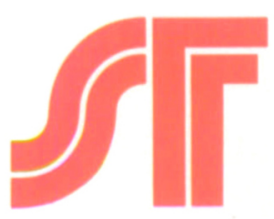

Sputtered Films Inc. 